\title{
Adding weight to the microbiota's role in obesity-exposure to antibiotics early in life can lead to increased adiposity
}

E xposure to antibiotics early in life can lead to increased body mass in humans and fat mass in mouse models, according to two new studies. The findings add further weight to the role of the gut microbiota in the development of obesity. "The hidden cost of antibiotics in early life may be its effects on host development, mediated via changes in the microbiome and its interaction with host cells," notes Martin Blaser (New York University, USA) who was involved in both studies.

Since their introduction in the early $20^{\text {th }}$ century, antibiotics have revolutionized medicine, substantially reducing infectionrelated morbidity and mortality. However, the ongoing misuse of antibiotics-for instance, overprescription and extensive use of low-dose antibiotics in livestock as growth promoters-have led to increasing concerns about the threat of antibiotic resistance and the long-term consequences of antibiotic exposure.

"I had known about farmers using low-dose antibiotics for growth promotion ... but asked the simple but serious questions-why does it work and how does it work?" explains Blaser. "If antibiotics early in life make farm animals bigger, could something analogous be happening to our children who are getting substantial antibiotic exposure to treat common infections," he questions.

In the first study, published in Nature, Blaser and co-workers used experimental models to explore how antibiotic exposure might affect host metabolism. Mice were exposed at weaning to subtherapeutic antibiotic treatment (STAT) - with either penicillin, vancomycin, penicillin plus vancomycin, chlortetracycline, or no antibiotic - at the mid-range of doses approved by the FDA for STAT in agriculture.

After 7 weeks of exposure, despite no changes in overall weight between all mice, dual-energy X-ray absorptiometry scanning revealed that total fat mass was

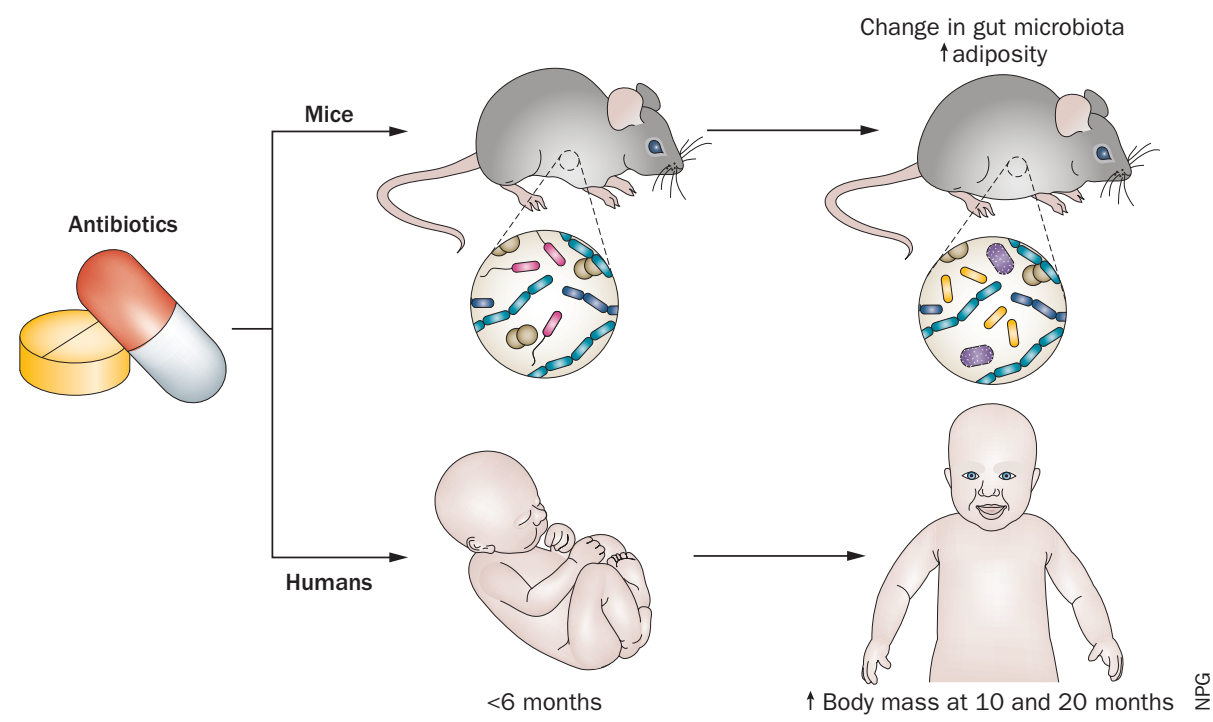

markedly higher in the STAT mice than in controls. Moreover, bone mineral density and levels of gastrointestinal inhibitory peptide-a hormone said to have a role in fatty acid metabolism via lipoprotein lipase activity-were also increased in STAT mice.

Investigating further, the authors found that STAT did not affect the overall microbe count in the mouse gut, but instead shifted the composition of the gut microbiota. STAT also led to changes in expression of genes involved in shortchain fatty acid metabolism and in the regulation of hepatic metabolism of lipids and cholesterol, as well as increased levels of colonic short-chain fatty acids.

To gain an epidemiological standpoint, data from 11,532 UK children enrolled in the Avon Longitudinal Study of Parents and Children were analysed in the second study. Whether the children received antibiotics was examined in three different time periods: $<6$ months, $6-14$ months and 15-23 months. Indices of body mass were determined at 6 weeks, 10 months, 20 months, 38 months and 7 years.

The findings, now published in the International Journal of Obesity, show that nearly one-third of the children received antibiotics by 6 months. Moreover, antibiotic exposure during this early time was associated with increased body mass (weight-for-length z-scores at 10 and 20 months) and BMI (at 38 months) during the child's development. Furthermore, infants exposed to antibiotics early in life were at markedly increased odds of being overweight as toddlers (at 38 months; OR 1.22; $P=0.029$ ); increased odds of obesity were also observed at 38 months, but were not statistically significant (OR 1.23; $P=0.097)$. Notably, the link between antibiotic exposure and body mass was observed even when accounting for obesity risk factors (such as an overweight mother or socioeconomic status).

The study authors acknowledge that further work is needed, but warn of the possibility of long-term metabolic consequences of antibiotic use early in life.

Katrina Ray

Original articles $\mathrm{Cho}$, I. et al. Antibiotics in early life alter the murine colonic microbiome and adiposity. Nature doi:10.1038/nature11400 | Trasande, L. et al. Infant antibiotic exposures and early-life body mass. Int. J. Obes. doi:10.38/ijo.2012.132 